\title{
Superresolution Differential Tomography: Experiments on Identification of Multiple Scatterers in Spaceborne SAR Data
}

\author{
Fabrizio Lombardini, Senior Member, IEEE, and Matteo Pardini, Member, IEEE
}

\begin{abstract}
Interest is growing in the application of coherent processing of synthetic aperture radar (SAR) data to the monitoring of complex urban or infrastructure areas. However, such scenarios are characterized by the layover phenomenon, in the presence of which conventional interferometric SAR techniques degrade or cannot operate. As a consequence, to monitor reliably a high number of ground structures, the identification, i.e., the detection and height and deformation velocity estimation, of both single and multiple scatterers interfering in the same SAR cell can be a key step. This issue is addressed here by means of differential tomography (Diff-Tomo), a recent multibaseline-multitemporal generalized interferometric framework which allows to resolve multiple moving scatterers at different heights in the same cell. In particular, superresolution adaptive Diff-Tomo is extensively tested and augmented with a new information extraction algorithm for the automated identification of the multiple scatterers. Experiments have been carried out with real C-band spaceborne data over urban areas; corresponding results are shown and discussed.
\end{abstract}

Index Terms-Detection, differential interferometry, multidimensional signal processing, synthetic aperture radar (SAR), tomography.

\section{INTRODUCTION}

I $\mathrm{N}$ THE past three decades, synthetic aperture radar (SAR) images have demonstrated unique capabilities for geoscience applications. To overcome the imagery misinterpretations due to the 3-D scattering projection onto the 2-D range-azimuth plane, cross-track SAR interferometry (InSAR) has been introduced. By exploiting the pixel-by-pixel phase variation between two or more SAR images acquired in viewing angle diversity (producing one or more interferometric baselines), InSAR has become a well-assessed technique for the generation of accurate digital elevation models (DEMs) [1]. However, such InSAR techniques do not take account of possible scatterer movements. For this reason, differential InSAR (D-InSAR) has been proposed based on the analysis of

Manuscript received November 1, 2009; revised March 5, 2010, June 11, 2010, November 22, 2010, and May 3, 2011; accepted June 19, 2011. Date of publication October 3, 2011; date of current version March 28, 2012. This work was supported in part in the framework of the Land and sea Integrated Monitoring for European Security (LIMES) project, Global Monitoring for Environment and Security (GMES) Sixth Framework Programme.

F. Lombardini is with the Department of Information Engineering, University of Pisa, 56122 Pisa, Italy (e-mail: f.lombardini@iet.unipi.it).

M. Pardini was with the University of Pisa, 56122 Pisa, Italy. He is now with the Microwaves and Radar Institute, German Aerospace Center (DLR), 82234 Oberpfaffenhofen, Germany (e-mail: matteo.pardini@dlr.de).

Color versions of one or more of the figures in this paper are available online at http://ieeexplore.ieee.org.

Digital Object Identifier 10.1109/TGRS.2011.2164925

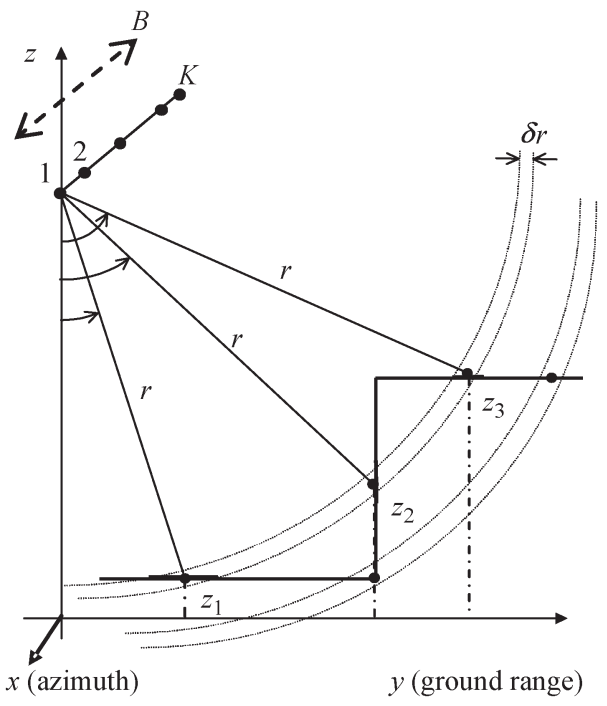

Fig. 1. Example of a MB acquisition geometry ( $K$ SAR images) over a scenario with three scatterers in layover at heights $z_{1}, z_{2}$, and $z_{3}$ in a first range-azimuth cell (denoted with an arc), and two scatterers in second cell at a larger range. $(x)$ Azimuth direction. $(y)$ Ground-range direction. $(z)$ Vertical height direction. $(r)$ Slant range. $(\delta r)$ Slant-range resolution. $(B)$ Total baseline.

temporal phase changes. In this context, D-InSAR stacking [2], persistent scatterer interferometry (PSI) [3], and the small baseline subset (SBAS) processing [4] are mature techniques based on multiple pass satellite SAR acquisitions. The possibility of accurately detecting and mapping centimeter- to millimeterscale deformations of the ground and of monitoring buildings, glacier flows, and slope instabilities with large coverage, high density of measures, and low cost has been widely demonstrated.

Nonetheless, the well-known layover phenomenon arises if the imaged area is characterized by the presence of a steep enough surface topography, generating critical projection of the scatterers in the slant imaging geometry, or by a high spatial density of strong scatterers. As a consequence, the signal collected in a SAR pixel may contain the superposition of the signals from multiple scatterers [1], [5], [6]. This condition is common also for volumetric scenarios such as forested areas, glaciers, and arid zones, particularly for low-frequency SARs, and it is frequent when data are acquired over complex scenarios such as urban areas or large structures and infrastructures. An example of a scenario with a step height variation (typical of urban areas) is shown in Fig. 1 in the ground-range-height plane, with three scatterers in layover in the first cell (denoted 
with an arc) and two scatterers in a second cell at a larger range. Existing InSAR and D-InSAR algorithms cannot separate the multiple-scattering phenomena in the same pixel, thus degrading or denying operation in these conditions.

Consequently, much interest has recently concerned the SAR 3-D tomography (Tomo-SAR) [7]-[18]. Tomo-SAR combines multibaseline (MB) acquisitions constituting a cross-track spatial array to achieve focused fully 3-D images through elevation beamforming (BF), i.e., spatial (baseline) spectral estimation, thus overcoming the limitation of standard InSAR techniques. Basically, Tomo-SAR can be regarded as a coherent (i.e., amplitude and phase) data combination technique in which the amplitude information is useful for exploiting the modulation induced by the beating phenomena to separate the multiple signals and to enhance the statistical accuracy even for single scatterers. In doing so, Tomo-SAR can add more features for solving InSAR height and reflectivity misinterpretation caused by layover geometries in natural or urban areas and for applications involving estimation of forest biomass and height, subcanopy topography, soil humidity, and ice thickness.

Although Tomo-SAR can separate multiple scatterers, it has no measuring sensitivity to their deformation motions in the line-of-sight direction. The first efforts to extend D-InSAR to the multiple-scatterer case have been recently proposed in [19] and [20]. In particular, the general framework of "differential tomography" (Diff-Tomo) in [19] handles jointly the spatial and the temporal dimensions deeply integrating the Tomo-SAR concept with the D-InSAR concept and fully exploiting the information content of $\mathrm{MB}$-multitemporal data with terrain movements. Joint resolution and estimation of the heights and the deformation velocity is thus allowed of the multiple scatterers in the same cell through a 2-D space-time "BF" in the height-velocity plane (i.e., 2-D baseline-time spectral estimation). Diff-Tomo-based techniques are thus powerful candidates for the analysis of layover urban scenarios.

Different processors have been proposed in the recent literature for Diff-Tomo analyses. To overcome the limited height-velocity resolution of the 2-D Fourier-based BF [19] and its poor sidelobe reduction capability, a deterministic approach was proposed in [21] and [22] in the framework of linear inverse problems. The Diff-Tomo image is obtained from the singular value decomposition (SVD) of the relevant linear imaging operator. However, even if the SVD can reduce the sidelobe amplitude, it does not have superresolution capabilities. Statistical methods have also been considered. The 2 -D version of the adaptive BF based on the Capon spectral estimator has been proposed in [19], together with the Fourierbased framework, and first tested with real data in [23]. Starting from a multilook estimate of the covariance matrix of the MB-multitemporal data vector, the adaptive Diff-Tomo BF has been demonstrated to possess the capabilities of superresolve scatterers in the height-velocity plane and reduce the sidelobe amplitudes. Recently, also the compressive sensing approach has been proposed for Tomo-SAR in [17] and applied to DiffTomo in [18].

This paper presents how superresolution adaptive Diff-Tomo, augmented with a scatterer number detector, can be employed for the automated extraction of the information about the height and deformation velocity of single and multiple scatterers in the same radar cell. More specifically, the contribution of this paper is threefold. First, the originator method of superresolution adaptive Diff-Tomo is improved beyond the first tests in [23] in order to handle automatically a large data set by augmenting it with a new original scatterer multiplicity detector for the extraction of the height/deformation velocity information. In particular, we tackle the detection problem by combining adaptive Diff-Tomo with a model-based least squares (LS) fitting in the complex (i.e., amplitude and phase) data domain. All the theoretical details and the discussion about the novelty of the proposed identification (i.e., detection and parameter estimation) algorithm are reported in Section II. Second, by means of the developed scatterer multiplicity detector, the extracted height/deformation velocity information is validated extensively, instead of on a number of selected cells. Third, a preliminary phenomenological analysis of the characteristics of the detected scatterers is performed, which is novel also considering the superresolution of the adaptive processing. These two points and the related results have been addressed in Section III by processing real C-band ERS-1/2 data over an area around the San Paolo stadium in the city of Naples and over the Cinecittà area in the city of Rome, with particular emphasis on single and double scatterers.

\section{Diff-Tomo Identification OF THE MULTiPle SCATterers}

This section is devoted to the theoretical presentation of the algorithm proposed for the scatterer identification. In Section II-A, the Diff-Tomo concept is recalled, and the relation is given linking the available MB-multitemporal data vector and the radar reflectivity function in the height-deformation velocity plane. Afterward, Section II-B presents the superresolution adaptive Diff-Tomo imaging algorithm, which constitutes the core technique used by the proposed scatterer identification method. Finally, Section II-C shows how the adaptive DiffTomo processor can be augmented with a new model-based 2-D baseline-time LS fitting in order to estimate automatically the number of scatterers interfering in the same radar cell.

\section{A. Diff-Tomo Concept}

The Diff-Tomo framework deeply integrates Tomo-SAR and D-InSAR, whereas the temporal dimension is neglected for Tomo-SAR, and only a single (possibly mean) height is extracted in conjunction with displacement measurements for D-InSAR. With reference to this latter technique, Diff-Tomo represents an extension of the D-InSAR scatterer separation concept in [20], in which the specific case of two layover scatterers with the same velocity is investigated.

We assume to process the data from $K$ repeated flight tracks of a single-channel SAR over the area of interest; multistatic SARs with multichannel acquisition capability [26] are available. After accurate (subpixel) calibration procedures, i.e., the 
coregistration of the images, the deramping operation, ${ }^{1}$ and the atmospheric propagation effect compensation, ${ }^{2}$ the focused signal collected in a generic range-azimuth pixel at the $k$ th track $(k=1, \ldots, K)$ can be written as [21], [22]

$$
[\mathbf{y}]_{k}=\iint \gamma(h, v)[\mathbf{a}(h, v)]_{k} d h d v
$$

where $\mathbf{y}$ is a $K$-dimensional data vector collecting the complex pixel amplitude at each track, $\gamma(h, v)$ is the unknown complex radar reflectivity function in the height-velocity domain, $h$ denotes the height, $v$ denotes the velocity, and $[\mathbf{a}(h, v)]_{k}$ is the $k$ th element of the so-called steering vector, defined as

$$
[\mathbf{a}(h, v)]_{k}=\exp \left[j 2 \pi\left(\frac{2 h}{\lambda R \sin \theta} B_{k}+\frac{2 v}{\lambda} T_{k}\right)\right] .
$$

In (2), $\lambda$ is the radar wavelength, $\theta$ is the look angle, $R$ is the slant range, $B_{k}$ is the $k$ th orthogonal baseline, and $T_{k}$ is the $k$ th acquisition time. Notice that, for a single-scattering component, (2) corresponds to a permanent scatterer response at the interferometric array [3]. It is apparent from (2) that the relation (1) between the received data at the different tracks and $\gamma(h, v)$ is a spatial-temporal spectral Fourier integral, with height-dependent spatial frequency

$$
\omega_{S}=\frac{2 h}{\lambda R \sin \theta}
$$

and velocity-dependent temporal frequency

$$
\omega_{T}=\frac{2 v}{\lambda} .
$$

In other words, each scattering component in the data originates a 2-D spatial-temporal harmonic from the data, and inversion of (1) is equivalent to a 2-D spatial-temporal spectral estimation. If the track distribution is uniform in the baseline-time plane, the reconstruction of $\gamma(h, v)$ can be simply obtained by a 2-D Fourier transform of the data. However, the distribution is generally far from being uniform, resulting in a very sparse 2-D sampling pattern. This sparsity produces intolerable 2-D sidelobes or quasi-grating lobes in the estimated height-deformation-velocity profiles. An example is shown in Fig. 2, which plots the point-spread function (i.e., the DiffTomo profile of a pointlike scatterer) of the data set over the city of Naples, with a peak sidelobe level around $-6 \mathrm{~dB}$. This aspect is peculiar to the Diff-Tomo imaging and requires the setup of advanced processing. For this reason, in this work, we make use of the 2-D version of the adaptive BF (indicated with $\mathrm{ABF}$ from here on for brevity) [19], which furnishes a leakage-reduced estimate of the backscattering intensity, i.e., of $P_{\gamma}(h, v)=E\left\{|\gamma(h, v)|^{2}\right\}$.

\footnotetext{
${ }^{1}$ The deramping compensates a phase term quadratic with the elevation along the baselines by using the orbital information and an available coarse DEM of reference. Moreover, the interferometric array constituted by the $K$ SAR acquisition positions is taken back to the convenient far-field behavior, furnishing a phase history which is linear with the orthogonal baselines. The deramping is effective only in a range of elevations around a reference one.

${ }^{2}$ The atmospheric phase calibration is carried out at low resolution by using specific multitemporal filtering procedures [10].
}

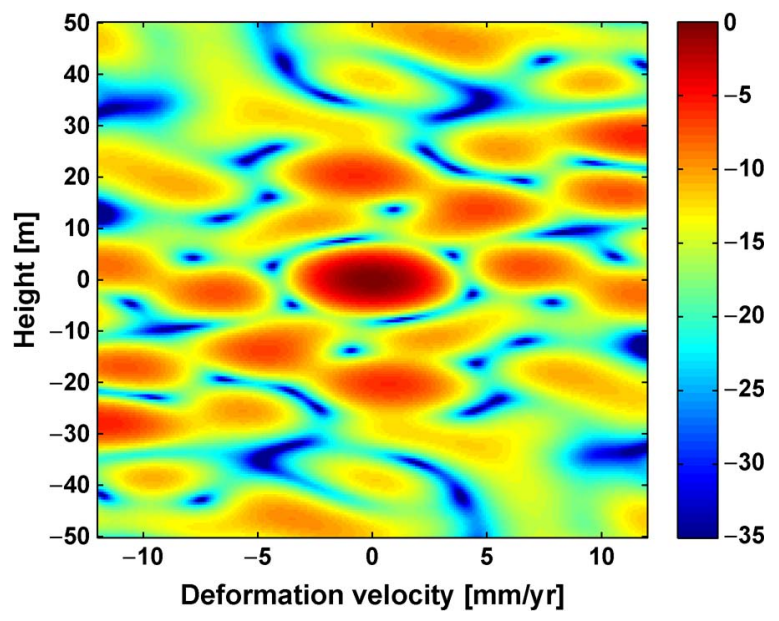

Fig. 2. Point-spread function (in decibels) of the ERS-1/2 data set over the city of Naples used for the real data experiments.

\section{B. Superresolution Diff-Tomo Processing}

The signal model (1) allows a reconstruction of the DiffTomo profile in the array signal processing framework [19], since a space-time distribution of equivalent phase centers of a "hybrid" planar array can be synthesized starting from the MB-multitemporal distribution of the radar platform passes in the cross-track plane. As a consequence, the application is enabled of 2-D spectral estimation methods to obtain an estimate of $P_{\gamma}(h, v)$. In particular, the Diff-Tomo ABF is nonparametric and belongs to the class of filterbank approaches for spectral estimation. Its expression can be derived by designing a finite impulse response of order $K$ that passes the spatial-temporal harmonic corresponding to the generic height $h$ and the velocity $v$ in the MB-multitemporal data vector $\mathbf{y}$ without distortion and, at the same time, attenuates as much as possible the power of noise and of other elevation-velocity components different from that of interest, making the produced 2-D response change depending on the input data during the height-velocity scan [19]. This behavior allows gains in terms of both resolution and leakage (sidelobe) level. ${ }^{3}$ The design criterion described before leads to the following expression for the filter coefficient vector:

$$
\mathbf{g}(h, v)=\frac{\hat{\mathbf{R}}^{-1} \mathbf{a}(h, v)}{\mathbf{a}^{H}(h, v) \hat{\mathbf{R}}^{-1} \mathbf{a}(h, v)}
$$

where $\hat{\mathbf{R}}$ is a multilook estimate of the covariance matrix of the data vector $\mathbf{y}$. The Diff-Tomo ABF estimate of the power spectrum $P_{\gamma}(h, v)$ is then obtained by calculating the power of the output of the filter (5).

We remark that vector $\mathbf{a}(h, v)$ in the processing is calculated given the orbital information and times in which the images have been acquired [10] with reference to the calibrated data. However, spurious phase contributions produced by residual

\footnotetext{
${ }^{3}$ The leakage level reduction is achieved by setting proper nulls in the produced spatial-temporal beam shape, thus rejecting the interference coming from scattering from other heights and velocities than the selected. In this sense, the spatial-temporal filter is data adaptive and exploits the information about the spatial-temporal power spectrum through the array covariance matrix.
} 
atmospheric effects and possible deramping errors can make the real form of the steering vector different from the nominal one (2). This mismatch can cause noticeable performance degradation, leading to the so-called signal self-nulling or selfcancellation, in which the useful signal at the $(h, v)$ coordinate of interest is seen as an interference and partially suppressed by the filter, instead of being left unaltered [27]. To mitigate these nonlinear radiometric effects and to avoid inversion problems of the covariance matrix due to the small number of looks compared with the number of tracks, a diagonal loading of $\hat{\mathbf{R}}$ can be included, with a total loading factor which is a multiple of the noise power estimate through a coefficient $\delta$ [9], [24]. In other words, the filter coefficients (5) are calculated by using $\hat{\mathbf{R}}+\delta \sigma_{W}^{2} \mathbf{I}$ in lieu of $\hat{\mathbf{R}}$, where $\sigma_{W}^{2}$ is the additive thermal noise power. However, there is not any simple rule for the choice of $\delta$, and it is usually determined empirically from a data analysis.

A second remark is in order. In addition to the mentioned improvements of $\mathrm{ABF}$ in terms of sidelobe rejection and superresolution with respect to the Fourier-based BF [23], a disadvantage is the need to implement a spatial multilooking that decreases the horizontal resolution of the height and velocity estimates of the detected scatterers. Nevertheless, the spatial multilooking is typically used also in polarimetric Tomo-SAR analyses of airborne urban data [14] and in the application of standard D-InSAR techniques, e.g., in the SBAS processing (for distributed scatterers) [4]. Anyway, for urban applications, single-look processing would be more desirable.

\section{Proposed Scatterer Detection Algorithm}

From a statistical point of view, at the $n$th look available for processing, the received signal vector in the presence of layover at the interferometric cross-track array can be modeled as a multicomponent $K$-dimensional complex-valued random vector as follows [5], [25]:

$$
\mathbf{y}(n)=\sum_{i=1}^{N_{S}} \sqrt{\tau_{i}} \mathbf{a}\left(h_{i}, v_{i}\right) \odot \mathbf{x}_{i}(n) \odot \mathbf{e}+\mathbf{w}(n)
$$

where $n=1, \ldots, N, N$ being the number of independent looks, $N_{S}$ is the number of scatterers, $h_{i}$ and $v_{i}$ are the height and the velocity, respectively, of the $i$ th scatterer, $\tau_{i}$ is the power of the $i$ th scatterer, and the symbol " $\odot$ " denotes the Schur-Hadamard product. Moreover, $\mathbf{x}_{i}(n)$ is a multiplicative noise modeling the speckle vector at the $n$th look and the $i$ th scatterer, possibly partially decorrelated in space (baseline decorrelation) and time. Vector $\mathbf{w}(n)$ is the thermal noise. Vector e contains the residual random miscalibration errors after atmospheric compensation and deramping; its $k$ th element can be written as $[\mathbf{e}]_{k}=\exp \left(j \phi_{k}\right)$, where the phases $\phi_{k}$ are commonly modeled as zero-mean random variables.

The detection of single scatterers (i.e., for $N_{S}=1$, absence of layover), with completely correlated speckle and, after the compensation (at least at a small scale) of the phase errors, has been first afforded in the PSI framework [3]. In that context, working with phase-only data, a scatterer is declared persistent if the mismatch between its phase history and the corresponding steering vector is below a threshold selected according to the experimental evidence. Recently, working in a coherent data combination framework, other techniques have been proposed based on the generalized likelihood ratio test [28].

In the case of multiple speckled scatterers (i.e., $N_{S}>1$ ), possibly corrupted by phase errors, the detection problem becomes atypical. A first contribution has been given in [20], even if it is limited to nondecorrelating double scatterers with the same velocity. In the multicomponent signal processing area, the classical detection methods are based on the information theoretic criteria (ITC), which rely on the eigenvalue distribution of the data covariance matrix. ITCs were originally conceived to operate with additive white Gaussian noise only (i.e., in the absence of multiplicative noise and phase errors); therefore, in a Tomo-SAR context, they are subjected to operate under a model mismatch, with a possible performance degradation. Nevertheless, their use has been investigated in ideal conditions in [29] and experimented in [12] by processing a spaceborne data set with reduced temporal decorrelation. Although reasonable results were obtained, the main concern with this "quick-look" detection technique is that it does not furnish a quality index on the estimated number of scatterers.

A suboptimal, but effective, detection algorithm was proposed in [30]. This technique combines adaptive tomography and a model-based fitting in the complex data domain for the estimation of the reflectivity of a number of hypothetical scatterers, which are then thresholded to obtain an estimate of $N_{S}$. The simulated analysis reported in [30] shows that this algorithm can outperform the ITC methods also in their regularized version. Recently, this algorithm has been extended to the case of the presence of data nonidealities and experimented on real data in [24] and [25], with a preliminary validation of the results obtained. A different algorithm has been presented in [31] and extended to the Diff-Tomo framework in [32]. It is based on a spectral model fitting in which the scattering distribution in the height-velocity plane is compared with the response to an ideal pointlike scatterer.

The detection algorithm presented here for scatterer identification extends the detection algorithm proposed in [25] to the Diff-Tomo framework, i.e., it combines superresolution adaptive Diff-Tomo processing with a 2-D model-based fitting in the complex data domain. Owing to the superresolution properties of the core adaptive Diff-Tomo processor, the proposed detector is expected to identify and locate a high number of layover scatterers at a height difference below the Rayleigh resolution limit. It is worth noting that experimental scatterer superresolution was neither claimed nor demonstrated at a large scale in the previous literature about scatterer separation [18], [20], [32]. The novelties of the proposed scatterer detector with respect to the ones existing in the literature are four. First, it is flexible, as it can handle easily the detection of a number of scatterers higher than two, which is different from [20] and [32]. In addition, different from the approach in [20], the detectable scatterers are not hypothesized to be pointlike, and no assumptions have been made about their relative velocity. Moreover, the proposed detector requires a simple setting of 
the thresholds, which are only two (on the estimated scatterer $S N R$ and on a properly defined fitting error) independently of the order, in contrast with the approach in [32], in which the number of thresholds to be set amounts to four to detect a double scatterer and it is expected to increase if the algorithm is extended to detect higher scatterer multiplicities. Finally, the computational complexity of the proposed detector is lighter with respect to both the approaches in [20] and [32], since the test statistics can be calculated in closed form for each model order and height and velocity estimates are directly extracted from the peaks of the Diff-Tomo ABF spectrum, which is calculated only once. Conversely, in [20], a multidimensional nonconvex search is needed for the nonlinear LS estimation of all the unknown parameters. On the other hand, the detection of double scatterers is performed in an iterative fashion in [32], and an SVD spatial-temporal spectrum is calculated two times at each iteration.

The detection of the scatterers is carried out by means of a sequential test. At the generic step, the presence of $M$ hypothetical scatterers is tested, with $M$ starting from one. Their heights and velocities are estimated from the locations of the $M$ highest peaks of the Diff-Tomo ABF spectrum. Notice that the calculation of the 2-D ABF spectrum is not needed at each step, resulting in a nonnegligible saving of computational burden. These estimates are then conveyed to a 2-D model-based LS fitting for the estimation of the corresponding scatterer powers according to the following functional (see [33] for the 1-D version):

$$
\hat{\boldsymbol{\alpha}}(n)=\arg \min _{\boldsymbol{\alpha}(n)}\|\mathbf{y}(n)-\mathbf{A} \boldsymbol{\alpha}(n)\|^{2}, \quad n=1, \ldots, N
$$

where $\boldsymbol{\alpha}(n)$ is an $M$-dimensional vector, whose elements are the scatterer complex amplitudes at the $n$th look, and $\mathbf{A}$ is the steering matrix whose columns are the steering vectors calculated for the $M$ heights and velocities estimated from ABF (denoted with $\hat{h}_{m}$ and $\hat{v}_{m}, m=1, \ldots, M$, respectively, in the following). Once the complex amplitudes are estimated from the fitting (7), the scatterer reflectivity estimates $\hat{\tau}_{m}$ are computed through the averaging of the squared absolute values of the amplitudes. It is worth remarking that the employment of the LS fitting is crucial to make the overall algorithm robust to the peak self-cancellation phenomenon affecting the ABF. Moreover, the functional (7) is model based since it is designed for the estimation of the power backscattered from pointlike targets, and it is statistically efficient only when white Gaussian noise is present. Nevertheless, the simulated analysis in [25], [30], and [33] has shown that the mismatch between (6) and (7) due to the presence of noncompact scatterers is generally not of concern. Finally, the signal-to-noise ratio associated to each hypothetical source is estimated as

$$
S \hat{N} R_{m}=\frac{\hat{\tau}_{m}}{\hat{\sigma}_{W}^{2}}
$$

Aside from the estimation of the signal-to-noise ratio, a normalized fitting error $\varepsilon(M)$ is calculated as well after solving

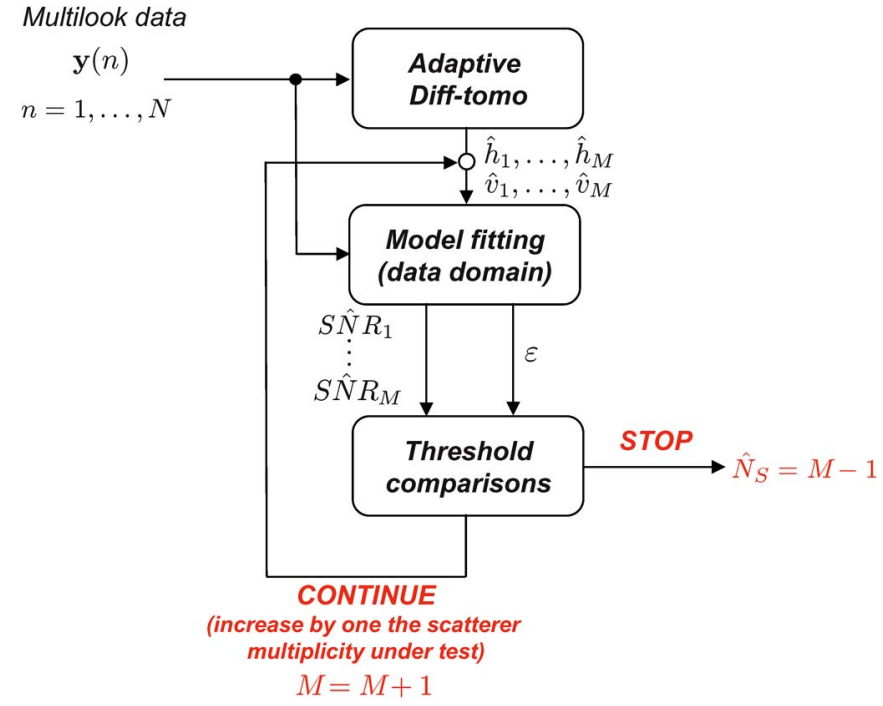

Fig. 3. Block scheme of the proposed scatterer multiplicity detector. Refer to Section II-C for detailed comments.

(7) defined as the ratio between the multilook averaged power of the fitting residuals and the total data power, i.e.,

$$
\varepsilon(M)=\frac{\sum_{n=1}^{N}\left\|\mathbf{y}(n)-\sum_{m=1}^{M} \hat{\alpha}_{m}(n) \mathbf{a}\left(\hat{h}_{m}, \hat{v}_{m}\right)\right\|^{2}}{\sum_{n=1}^{N}\|\mathbf{y}(n)\|^{2}}
$$

where $\hat{\alpha}_{m}(n)$ is the estimate of the complex amplitude of the $m$ th hypothetical scatterer, obtained from (7), at the $n$th look (i.e., the $m$ th element of vector $\hat{\boldsymbol{\alpha}}(n), m=1, \ldots, M)$.

Both the $S \hat{N} R$ 's and $\varepsilon(M)$ are thresholded to control the iterations and output the estimated number of scatterers. For the sake of clearness, a block scheme of the detection algorithm is shown in Fig. 3. In ideal conditions, a simple yet effective strategy proceeds blockwise (i.e., it estimates the $S N R$ of an assumed maximum number of sources) and declares that a scatterer is present if the corresponding $S \hat{N} R_{m}$ is higher than a threshold; otherwise, it is classified as a noise component. In [30], this criterion has been demonstrated to be sufficient to outperform ITC-based methods. However, when data miscalibration and signal decorrelation are jointly present, a situation which typically occurs with real data, it can be observed that, for the tested model order exceeding the correct one, the false-scatterer $S \hat{N} R$ increases, whereas the $S \hat{N} R$ of the true scatterers decreases. To visualize this phenomenon, we simulated the signal received from two elevation compact scatterers in layover according to model (6) with the baseline-time pattern of the 30-track Naples data set used for the real data experiments (see Section III for a brief description), with a distance of $10 \mathrm{~m}$ in vertical height, which is slightly larger than the Rayleigh resolution limit $(8.8 \mathrm{~m})$, and equal velocity. We generated 100 Monte Carlo realizations of the multilook signal vectors, for different values of the $S N R$ of one scatterer, while the $S N R$ of the other was kept fixed to $12 \mathrm{~dB}$. We then estimated the reflectivities of five hypothetical scatterers, and we calculated the corresponding $S N R$. Fig. 4(a) shows the 


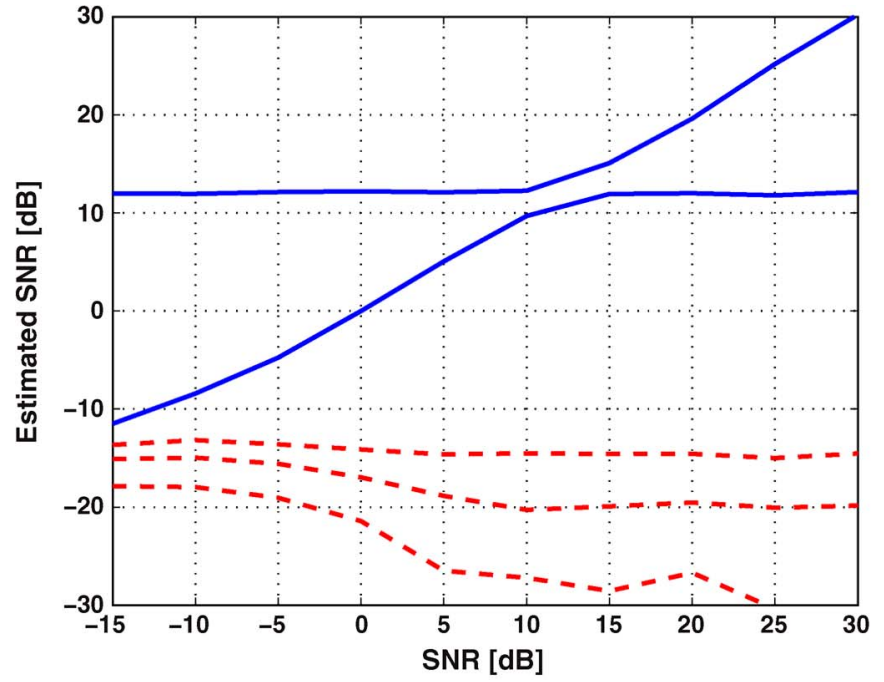

(a)

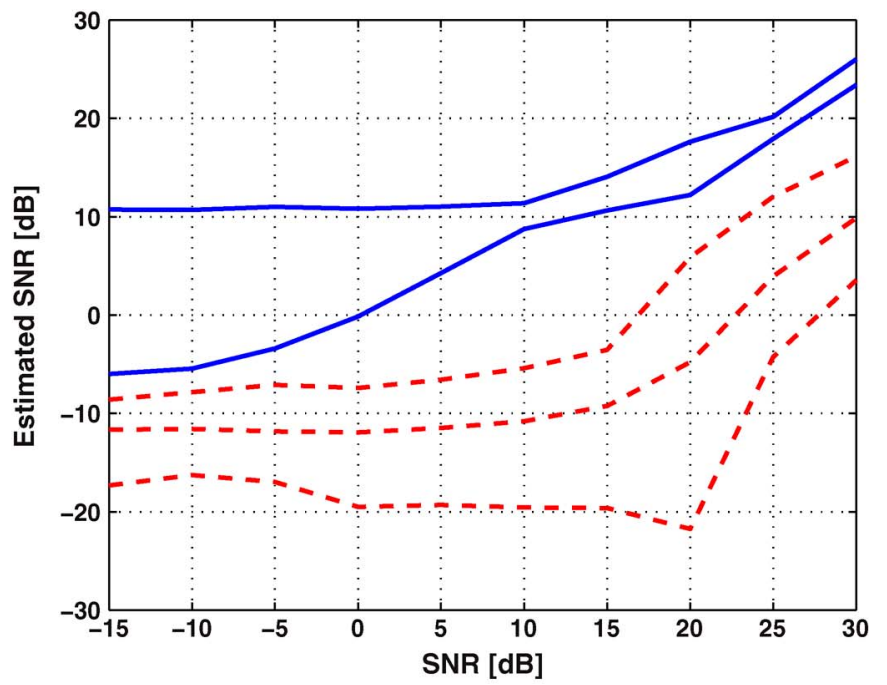

(b)

Fig. 4. Mean values of the estimated $S N R$ 's of five hypothetical scatterers in the presence of miscalibrations, with $N_{S}=2$ as a function of the $S N R$ of one scatterer. (Blue continuous line) True scatterers. (Red dashed lines) Other scatterers (overfitting). (a) Ideal case. (b) In the presence of residual miscalibration and a slight baseline decorrelation.

mean value of the five estimated $S N R$ 's as a function of the varying $S N R$ in the absence of miscalibration. The estimated $S N R$ of the false (overfitted) scatterers remains constant and lower than $-14 \mathrm{~dB}$, thus allowing to set a low threshold on the $S \hat{N} R$ for high-performance detection, as in the algorithm in [30]. When a residual phase miscalibration and a slight baseline decorrelation are introduced to corrupt the data, the curves in Fig. 4(b) are obtained. The estimated SNR's of the false scatterers increase noticeably, particularly for high $S N R$. As a companion effect, the mean estimated $S N R$ of the true scatterers can be slightly reduced with respect to the true value. The observed behavior is tantamount to conclude that the presence of miscalibrations makes $S \hat{N} R$-only thresholding become very challenging, resulting in increased probability of false alarm or low detection sensitivity.

A possible remedy to keep the number of false alarms low (at least at middle-high signal-to-noise ratios, generally observed in urban scenarios) is the thresholding of the fitting error $\varepsilon(M)$. In fact, $\varepsilon(M)$ does not approach zero with the correct model order (as in the ideal case), and it decreases further when the hypothesized scatterer multiplicity $M$ becomes higher than the correct one. This is due to the fact that components of signal perturbations from the data nonidealities are fitted as if they were from true scatterers [25]. This phenomenon is exploited in the proposed sequential algorithm, i.e., $\varepsilon(M)$ must not be below a certain threshold; otherwise, the condition of overfitting is declared by the test. In conclusion, at the generic $M$ th step, the LS fitting (7) is carried out fed by $\hat{h}_{1}, \ldots, \hat{h}_{M}$ and $\hat{v}_{1}, \ldots, \hat{v}_{M}$ extracted from the ABF spectrum, and the $S \hat{N} R$ 's $\left(S \hat{N} R_{1}, \ldots, S \hat{N} R_{M}\right)$ and the fitting error $\varepsilon(M)$ are compared to their respective thresholds. If both comparisons are positive (i.e., all the hypothetical scatterers have enough $S \hat{N} R$ and the overfitting is not declared), then the algorithm tests the presence of $M+1$ scatterers; otherwise, it outputs the presence of $M-1$ scatterers. In other words, the detector outputs the last tested order corresponding to significant components without overfitting. The thresholds are set by a data analysis over selected scatterers.

\section{DifF-Tomo Results}

In this section, the results obtained from experiments carried out with simulated and real data are presented. In particular, the real data experiments have been carried out by processing C-band spaceborne ERS-1/2 data, with different phase calibration qualities. Two MB-multitemporal data sets have been considered over a suburban area in the city of Naples (Italy) around the San Paolo stadium. A third data set has been processed over the "Cinecittà" area in the city of Rome (Italy). For each data set, before carrying out the tomographic processing, the images have been coregistered precisely. Then, exploiting an external DEM, the deramping operation has been performed, steering the MB array to the local topography, which is also the reference of the estimated heights. Finally, the phase distortions due to the atmospheric screen [3] have been removed by means of a low-resolution processing [10], [24]. These calibration steps have been carried out by Istituto per il Rilevamento Elettromagnetico dell'Ambiente-Consiglio Nazionale delle Ricerche (IREA-CNR). The first data set over Naples is composed of 30 images covering a time span of approximately six years and three months (1992-1998), corresponding to a line-of-sight velocity resolution of $4.5 \mathrm{~mm} /$ year. The overall orthogonal baseline measures $1066 \mathrm{~m}$, which furnishes a vertical height Rayleigh resolution limit of $8.8 \mathrm{~m}$. Baseline distribution and acquisition times are reported in [10]. The second data set over Naples is composed of 63 images acquired between 1992 and 2004, with an overall baseline of $1700 \mathrm{~m}$, a height resolution limit of $5.5 \mathrm{~m}$, and a velocity resolution of approximately $2.3 \mathrm{~mm} /$ year. The Rome data set is composed of 40 images acquired between 1995 and 2000, with an overall baseline of $1500 \mathrm{~m}$ and a height resolution of $6.4 \mathrm{~m}$. The time span of about five years leads to a velocity resolution of $5.6 \mathrm{~mm} /$ year. All of the three data sets have a slant-range resolution equal 


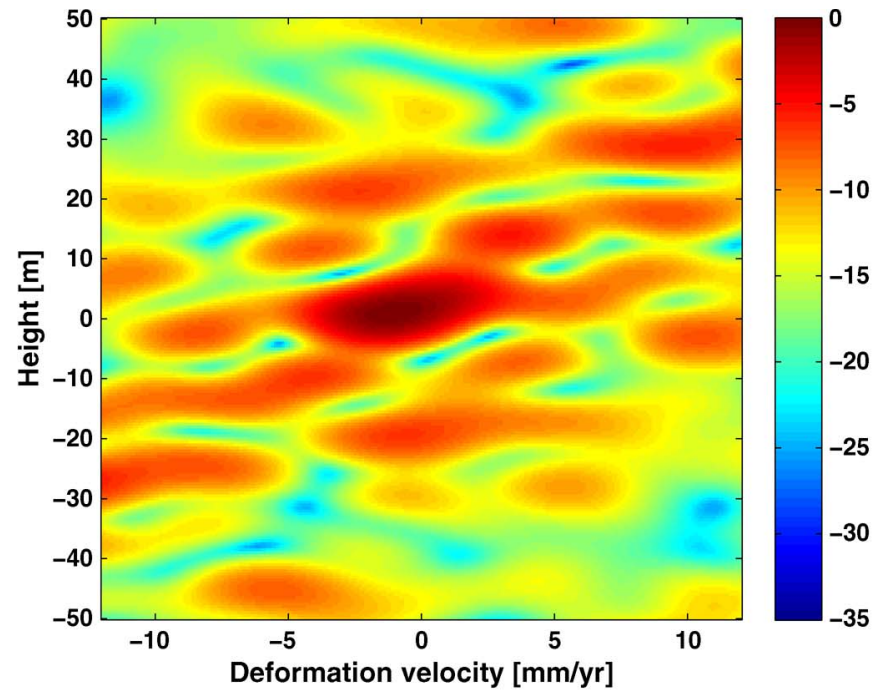

(a)

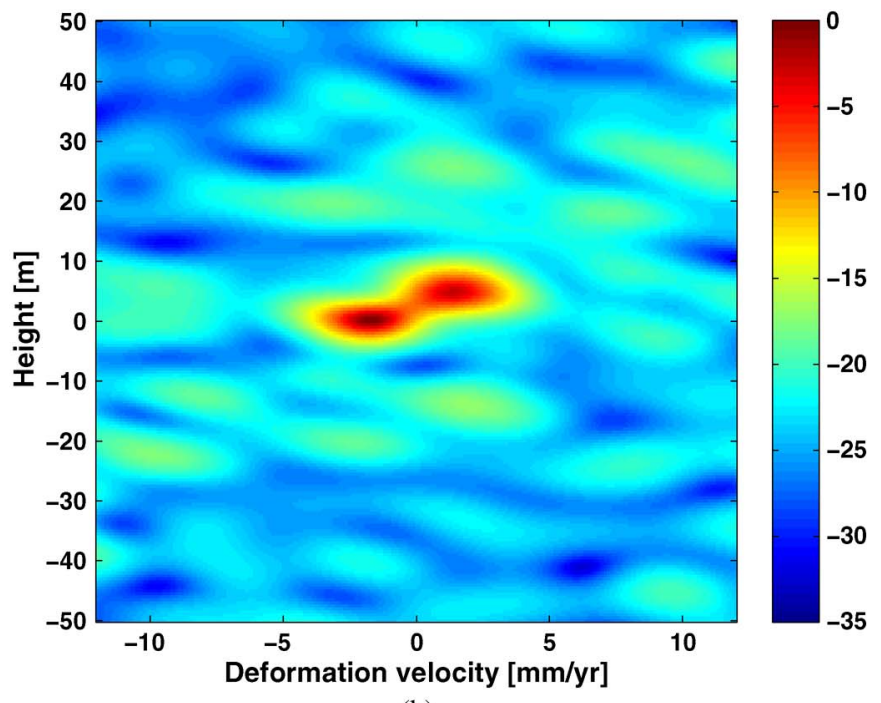

(b)

Fig. 5. Estimated Diff-Tomo spectra (in decibels) of two simulated pointlike targets at a height difference below the Rayleigh resolution limit and with a relative motion. (a) Diff-Tomo BF. (b) Diff-Tomo ABF.

to $9.6 \mathrm{~m}$ (corresponding to $24.7 \mathrm{~m}$ in ground range) and an azimuth resolution equal to $5.6 \mathrm{~m}$. Experiments have been carried out by averaging five adjacent pixels in the azimuth direction, the resulting multilook cell projected onto the ground being almost a square, with a side measuring about $25 \mathrm{~m}$. It is worth noting that the 63-track Naples and the Rome data sets are calibrated in phase with more refined procedures; the effect in the velocity and height estimation will be further commented upon analysis of the results.

\section{A. Simulated Analysis}

To get a flavor of the potentialities of the Diff-Tomo $\mathrm{ABF}$ processing, a simulated analysis has been carried out. Considering the baseline-time pattern of the 30-track data set, the presence of two pointlike scatterers with a relative motion of $4 \mathrm{~mm} /$ year has been simulated according to the signal model (6), in the absence of random phase residuals, with a total signal-to-noise ratio of $12 \mathrm{~dB}$. The height difference amounts to $6 \mathrm{~m}$, which is below the Rayleigh resolution limit for this data set. The Diff-Tomo profile estimated with the Fourierbased BF is shown in Fig. 5(a), normalized with respect to its maximum and rescaled in decibels. Apart from the presence of inflated sidelobes, the two scatterers are not distinguishable. Conversely, by employing the ABF spectral estimator [Fig. 5(b)], the two scatterers are perfectly separated, and their heights and velocities can be extracted. Moreover, with $\mathrm{ABF}$, it is possible to obtain a peak sidelobe level of $-15 \mathrm{~dB}$, which is $10 \mathrm{~dB}$ better than that achievable with BF. We remark that, with a Tomo-SAR focusing of the same data vector, a misinterpretation of the baseline signal histories (i.e., related to scatterer heights) with the temporal histories (i.e., related to the scatterer deformation) would occur. As a consequence, even the extraction of the scatterer heights would have been impossible with a height-only focusing.

\section{B. Real Data Results of Scatterer Identification}

The test area over Naples is located around the San Paolo stadium. This area measures about $1.5 \mathrm{~km}$ in ground range

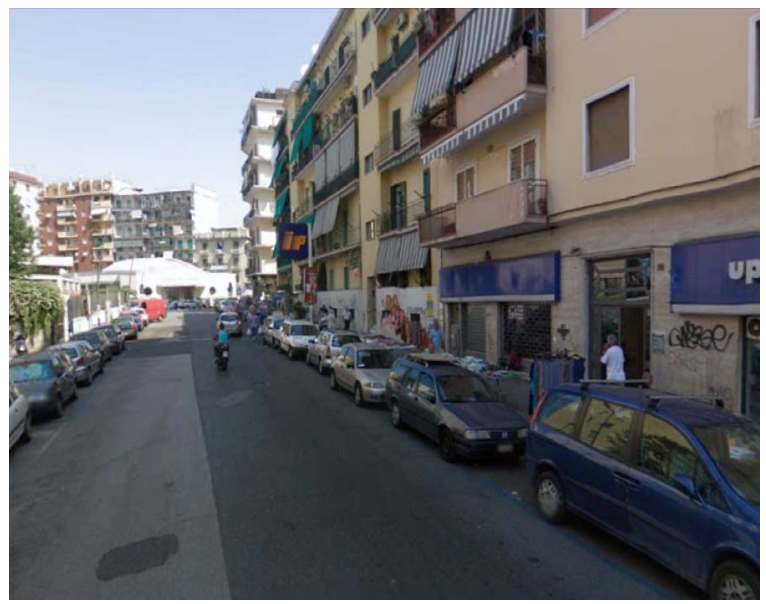

Fig. 6. Photograph captured with Google Street View of the typical buildings present in the test area of the Naples data set.

and about $5.5 \mathrm{~km}$ in azimuth, and it is a part of the whole data set, which covers nearly $8.1 \mathrm{~km}$ in ground range and the same extension in azimuth. The area surrounding the stadium is composed mainly of buildings of three/four floors or even more, of which an example is shown in the picture in Fig. 6, and other industrial buildings and infrastructures. Also, vegetated areas are present. An aerial photograph of the entire test area is shown in Fig. 7(a); the San Paolo Stadium is the elliptical structure approximately at the center of the image. The proposed automated scatterer identification method has been applied over the whole scene; zones with low coherence have not been processed. The coherence has been estimated by averaging 11 azimuth pixels; this number has been chosen from an empirical tradeoff analysis, taking into account precision of the estimation and homogeneity of the multilook cell. In order to obtain a reliable estimation of the coherence, we selected an image pair with, at the same time, the shortest possible spatial baseline and a time span covering at least half of the total time span of the acquisitions. A very short baseline is needed to minimize as much as possible the baseline decorrelation 


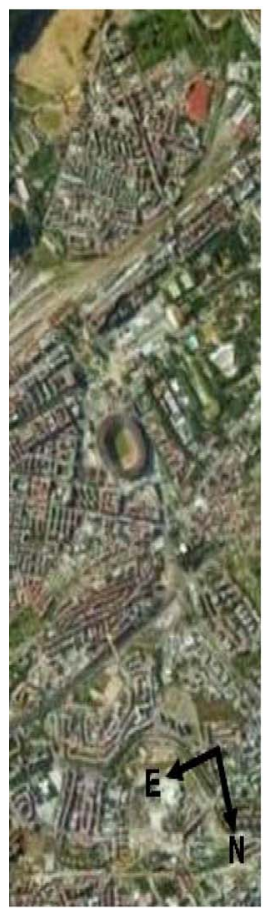

(a)

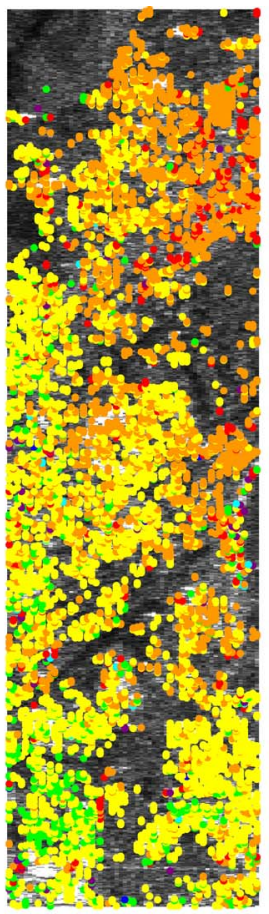

(b)

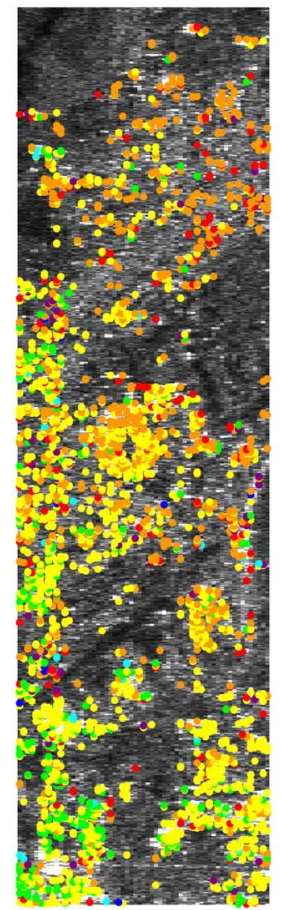

(c)

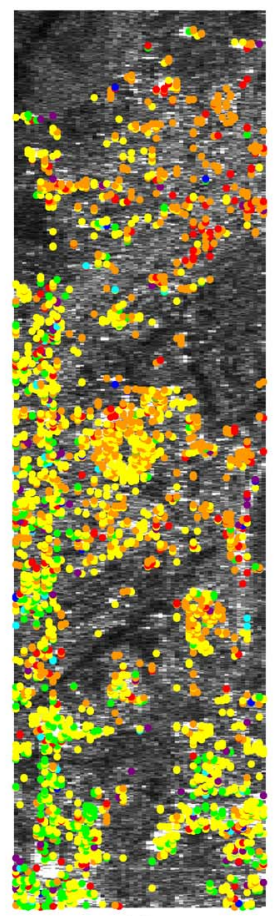

(d)
$>0.6 \mathrm{~cm} / \mathrm{yr}$
$0.4: 0.6 \mathrm{~cm} / \mathrm{yr}$
$0.2: 0.4 \mathrm{~cm} / \mathrm{yr}$
$-0.2: 0.2 \mathrm{~cm} / \mathrm{yr}$
$-0.4:-0.2 \mathrm{~cm} / \mathrm{yr}$
$-0.6:-0.4 \mathrm{~cm} / \mathrm{yr}$
$<-0.6 \mathrm{~cm} / \mathrm{yr}$

Fig. 7. Color-coded deformation-velocity estimates of the identified single scatterers with the 30-track data set, superimposed to the MB-averaged single-look ERS radar image. Range direction is on the horizontal axis, and azimuth direction is on the vertical one. (a) Google Earth image of the test area. (b) Estimated velocities of the identified single scatterers. (c), (d) Estimated velocities of the identified double scatterers (dominant and secondary, respectively).

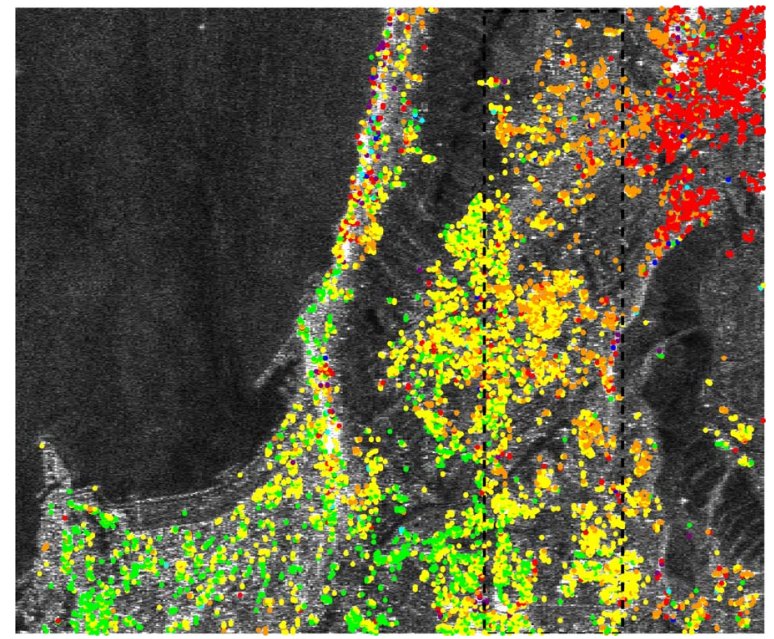

Fig. 8. Deformation-velocity estimates of the identified dominant double scatterers over the whole area (30-track data set), superimposed to the radar image. The dashed rectangle indicates the test area. The color coding is the same as that in Fig. 7.

induced by the presence of interfering signals coming from multiple (possibly extended) scatterers in the same cell. In doing so, the observed coherence is mainly due to temporal effects. Concerning the two data sets of Naples, the coherence has been estimated with a baseline of about $10 \mathrm{~m}$ and a temporal span of 5.5 years. In order to estimate the $S N R$ 's from the reflectivity estimates, the noise power was estimated on an independent area.

The estimated (unfiltered) velocities of single scatterers detected with the 30-track data set (the one with lower phase calibration quality) over the test area are shown in Fig. 7(b), coded with colors and superimposed on the radar image.

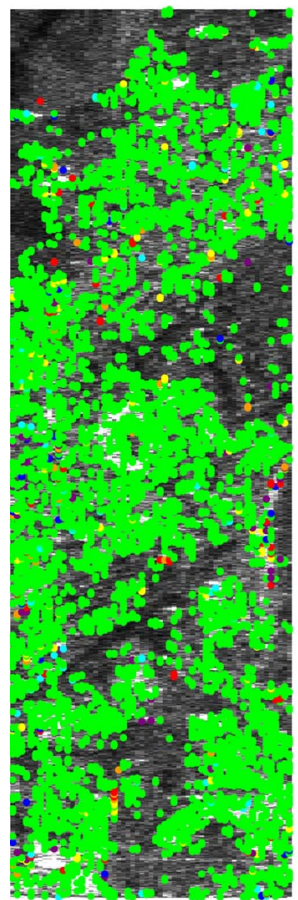

(a)

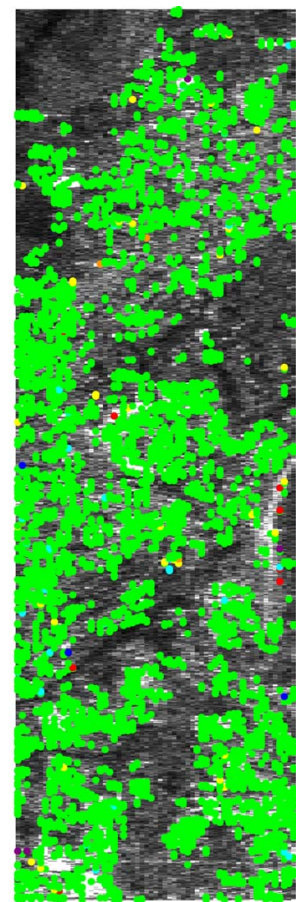

(b)
Fig. 9. Deformation-velocity differences of the detected single scatterers on the test area with respect to available independent D-InSAR measurements, superimposed to the radar image. The color coding is the same as that in Fig. 7. (a) Thirty-track data set. (b) Sixty-three-track data set.

Areas with homogeneous velocity distribution can be found. Moreover, it can be observed that the velocity progressively decreases passing from the bottom left to the top right parts of the test area where well-known subsidence phenomena are 


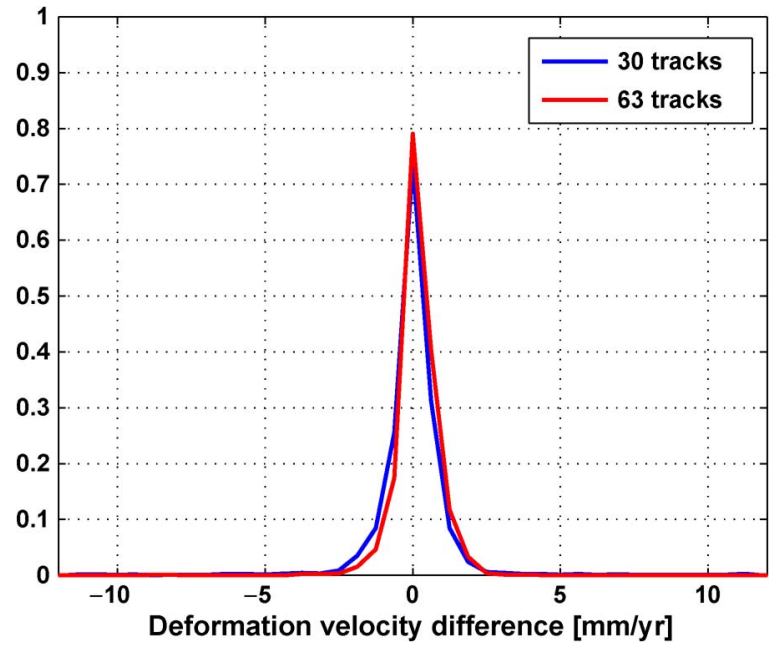

Fig. 10. Normalized histogram of the deformation-velocity differences shown in Fig. 9.

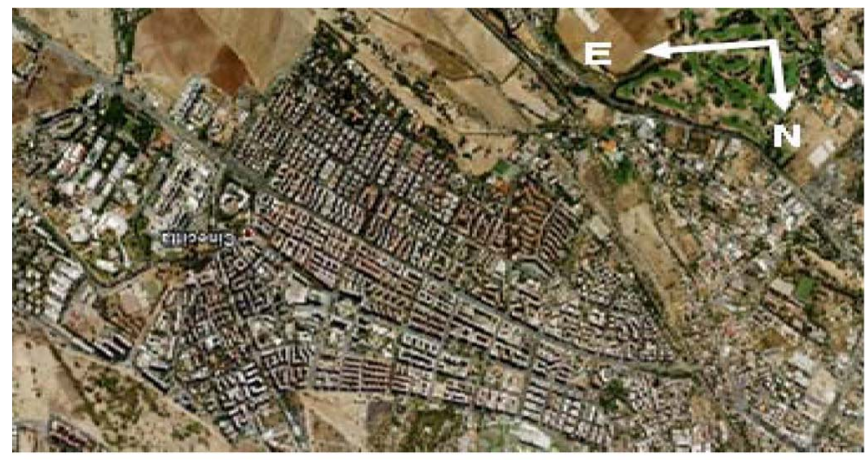

(a)

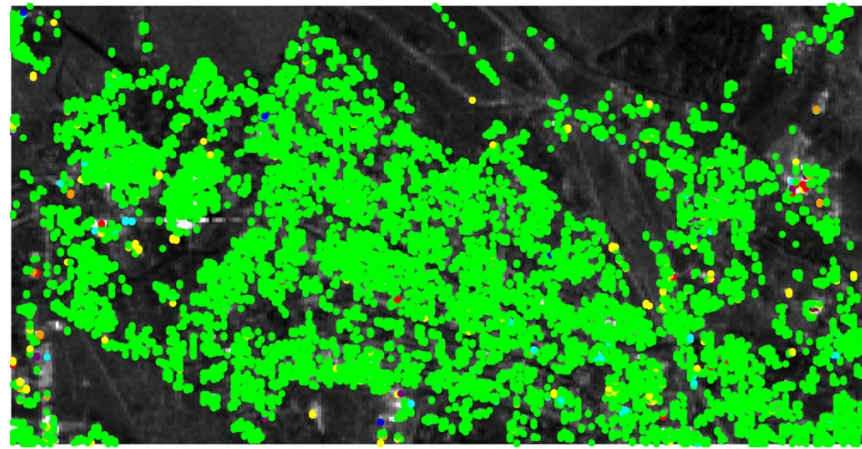

(b)

Fig. 11. Cinecittà area in Rome (Italy) and the estimated deformation-velocity differences with respect to available independent D-InSAR measurements of the detected single scatterers, superimposed to the radar image. Range direction is on the horizontal axis, and azimuth direction is on the vertical one. The color coding is the same as that in Fig. 7. (a) Google Earth image. (b) Estimated relative deformation-velocity map.

present. Fig. 7(c) and (d) shows the estimated velocities of the identified dominant and secondary (weaker) double scatterers, respectively. In Fig. 8, the velocities of the dominant double scatterers are shown over the entire area covered by the data set. The large-scale velocity behavior of the dominant double scatterers agrees with that of the secondary and of the single ones, as it is reasonable to expect. We also notice that the detected single scatterers are about 9500 , whereas the double scatterers are 3200 (33.5\% of the single ones). As a consequence, the

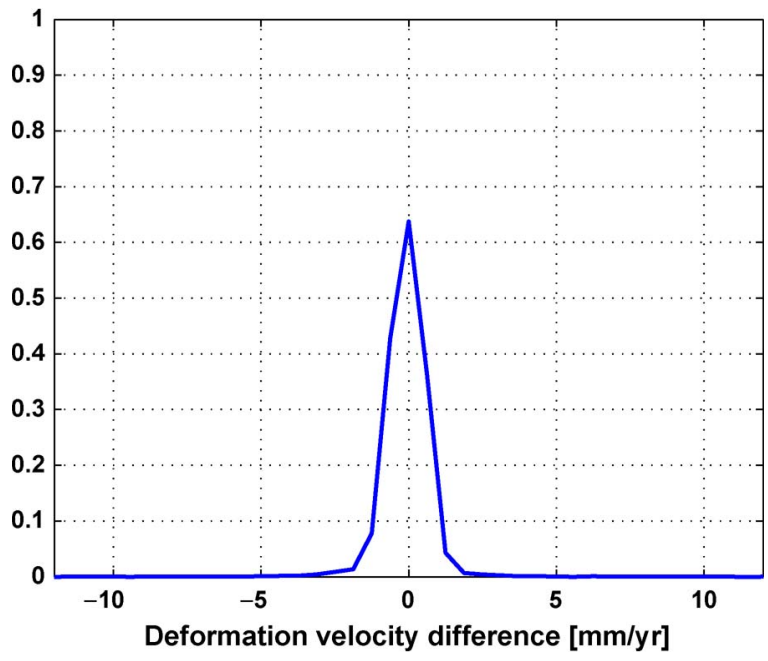

Fig. 12. Normalized histograms of the deformation-velocity differences shown in Fig. 11.

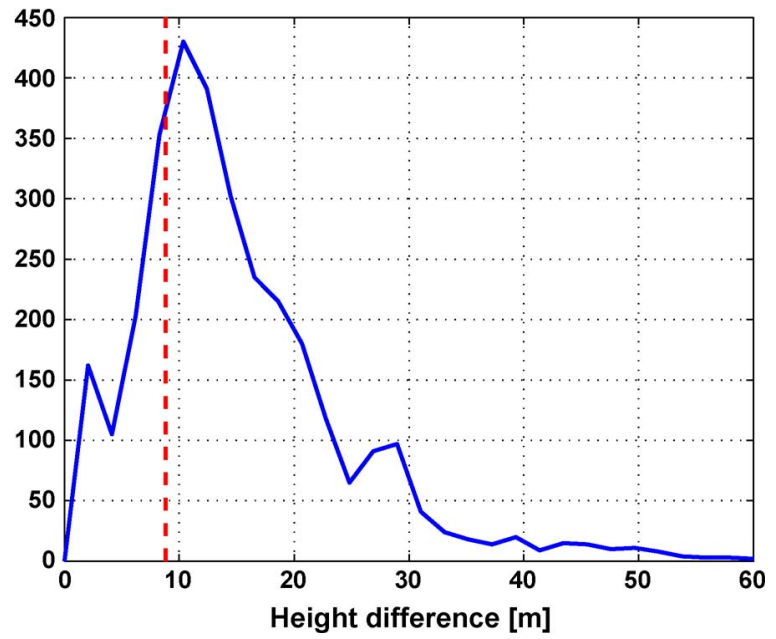

Fig. 13. Histogram of the height differences of the identified double scatterers with the 30-track data set. The vertical dashed line delimits the histogram region (on the left) corresponding to double scatterers with sub-Rayleigh height difference.

total number of velocity measures obtained with the double scatterers amounts to $67 \%$ of those corresponding to the single scatterers. A performance evaluation of the velocity estimation with respect to what is obtained with an operative D-InSAR technique is reported in the next section, also considering the better calibrated 63-track data set on the same area.

Scatterer identification has been carried out also with the Rome data set, which refers to a suburban area of about $5 \mathrm{~km}$ in ground range and about $2.5 \mathrm{~km}$ in azimuth. For this data set, the coherence has been estimated with a spatial baseline of $70 \mathrm{~m}$ and a temporal span of 2.5 years. In areas with medium-high coherence, we detected about 14300 single scatterers and 2500 double scatterers, i.e., around $17 \%$ of the single ones.

\section{Validation of the Extracted Velocity Measures}

As regards the data sets over Naples, the velocities of the single scatterers have been compared with the velocities obtained with the SBAS technique [4]. The velocity differences 


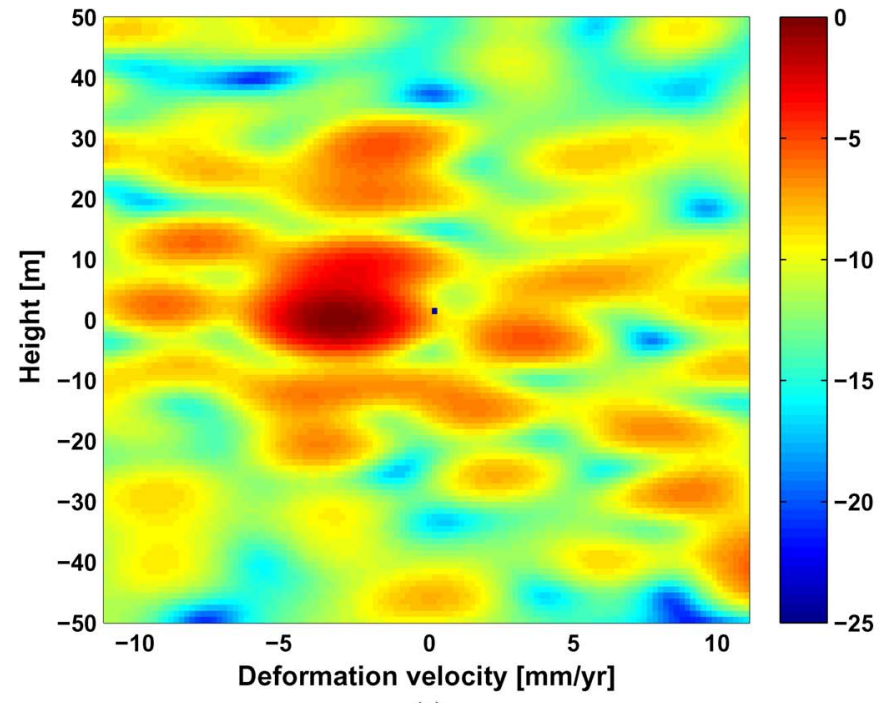

(a)

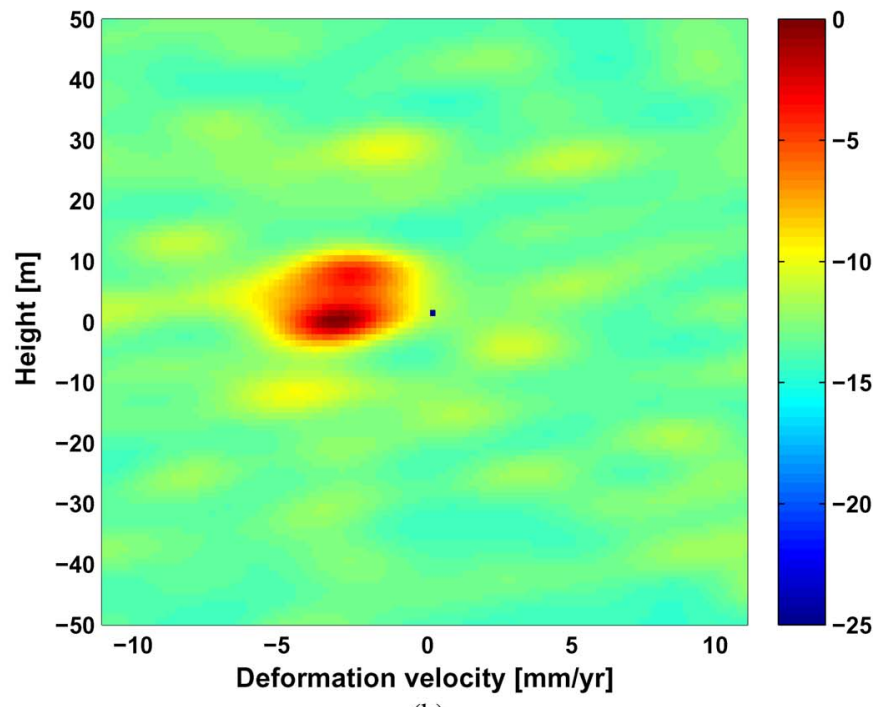

(b)

Fig. 14. Example of Diff-Tomo spectra (in decibels) of a double scatterer with sub-Rayleigh height difference on a flank of the San Paolo stadium detected with the 30-track data set. (a) Diff-Tomo BF. (b) Diff-Tomo ABF.

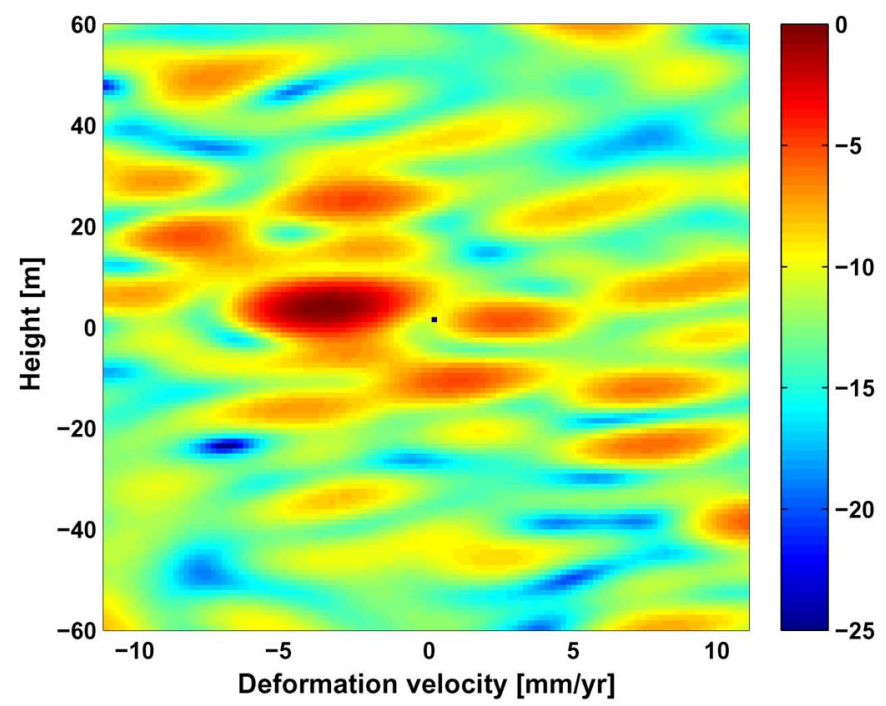

(a)

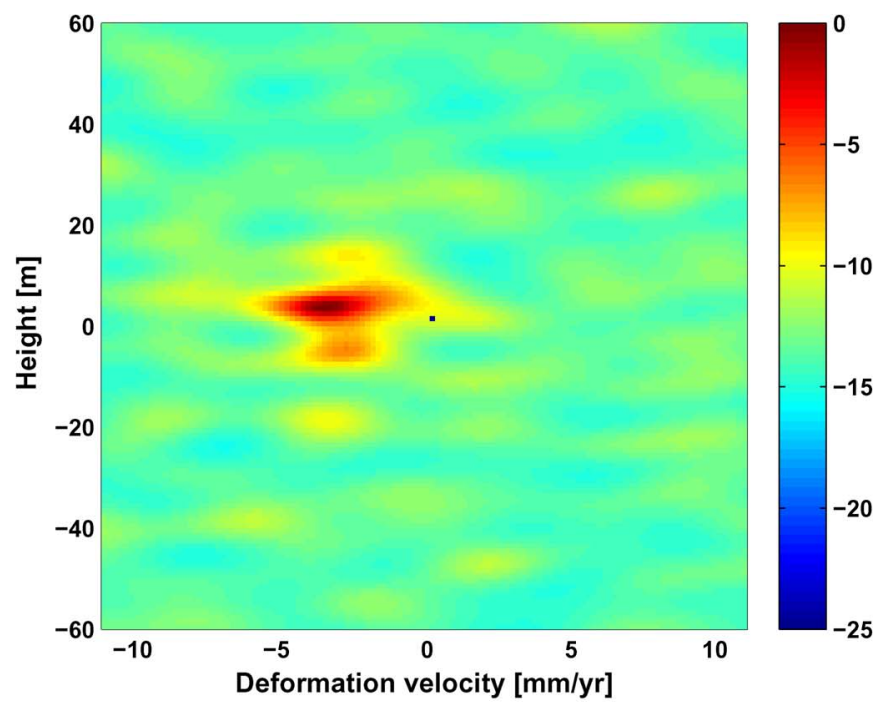

(b)

Fig. 15. Example of Diff-Tomo spectra (in decibels) of a double scatterer with sub-Rayleigh height difference on a building close to the San Paolo stadium detected with the 30-track data set. (a) Diff-Tomo BF. (b) Diff-Tomo ABF.

are mapped in Fig. 9(a) with the same color coding as that in Fig. 5, again superimposed to the radar image; the corresponding normalized histogram is plotted in Fig. 10 (blue curve). The standard deviation of the velocity difference is $1.4 \mathrm{~mm} /$ year, which makes the velocities estimated by the proposed automated Diff-Tomo processing reasonably consistent with those estimated by an operative D-InSAR technique. This analysis has also been carried out for the 63-track data set over the same area. The velocity difference map is shown in Fig. 9(b), and the corresponding normalized histogram is the red curve in Fig. 9. In this case, owing also to a more effective data calibration with respect to the 30-track data set given the more dense baseline-time sampling pattern, the standard deviation decreases to $0.7 \mathrm{~mm} /$ year.

The validation of the estimated scatterer velocities has been carried out on the data set over the city of Rome as well. In this case, we compared the estimated velocities with the available D-InSAR measures obtained with the recently developed enhanced spatial difference algorithm [24], [34]. In Fig. 11(a), an optical image of the area is shown; the map of the velocity differences is shown in Fig. 11(b), superimposed to the radar image, and the corresponding histogram is plotted in Fig. 12. The standard deviation of the velocity differences amounts to 1 $\mathrm{mm}$ /year, a low value obtained also owing to the more refined calibration procedures (see [24] and references therein).

\section{Height Superresolution Assessment and Phenomenological Analysis}

Jointly with the deformation velocities in Fig. 7, also the heights of the single and double scatterers have been estimated. In Fig. 13, the histogram of the height difference of the 


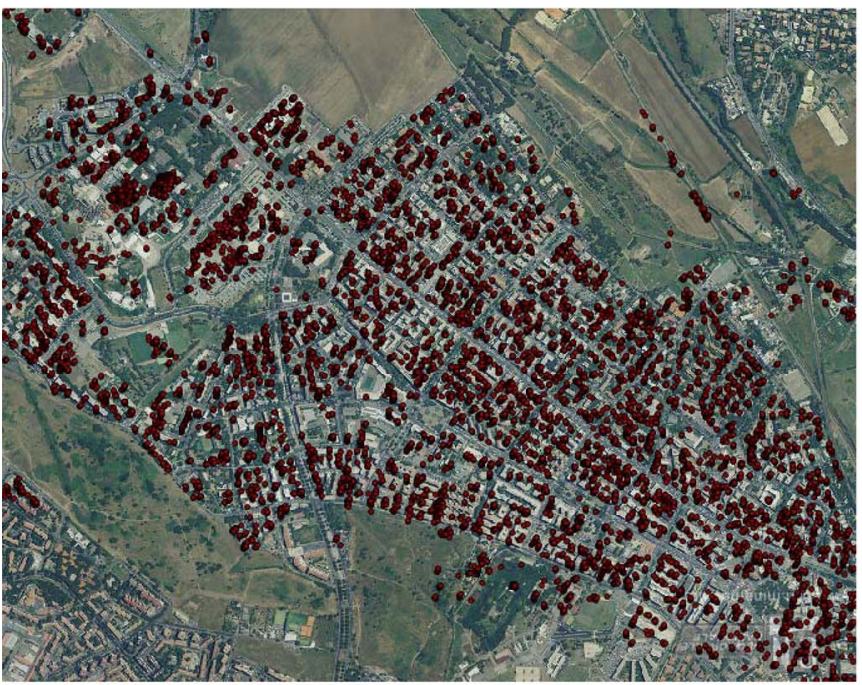

(a)

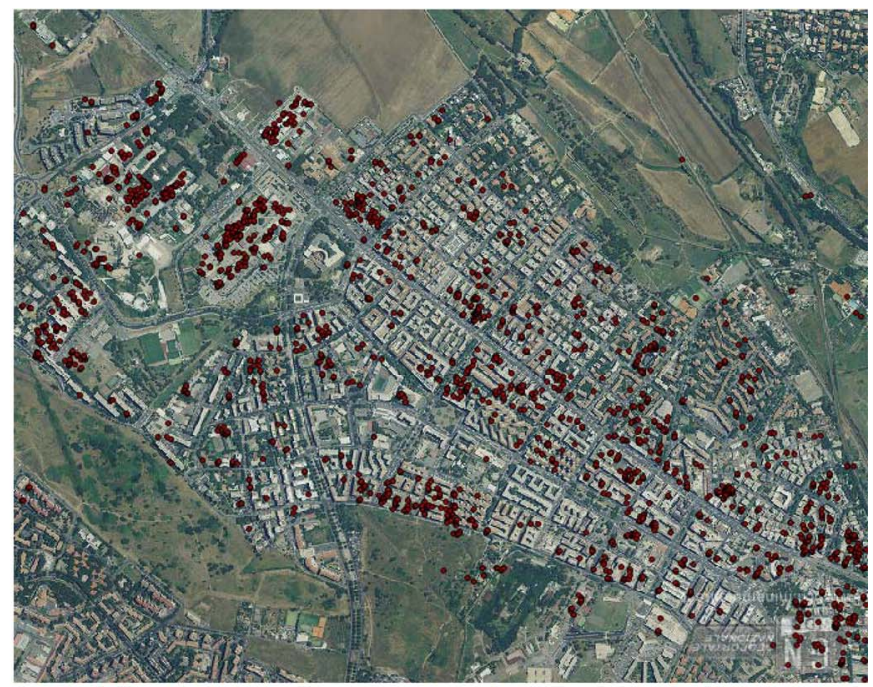

(b)

Fig. 16. (Dark red dots) Geocoded positions of the scatterers detected with the Rome data set. (a) Single scatterers. (b) Dominant double scatterers.

double scatterers is reported estimated with the 30-track data set over Naples. The mean height difference is around $15 \mathrm{~m}$, a reasonable value considering the kind of buildings on the test area (see also Fig. 5) and taking into account that a typical height difference between floors is $3 \mathrm{~m}$. It is apparent also that a number of double scatterers have been detected with a height difference below the Rayleigh resolution limit (8.8 $\mathrm{m}$ with this data set), owing to the superresolution properties of the ABF Diff-Tomo profiling. More precisely, they are about 550, i.e., about $18 \%$ of the double scatterers. Concerning the Rome data set, we found 470 double scatterers superresolved in height, i.e., $18 \%$ of the total number of the scatterer pairs.

The following results refer to the 30-track Naples data set. Examples of ABF Diff-Tomo profiles of two superresolved close-by targets are shown in Figs. 14 and 15, detected on a flank of the stadium and on a building in its neighborhoods, respectively. The corresponding BF Diff-Tomo profiles have been reported for comparison. The profiles are normalized with respect to their maxima and rescaled in decibels for easiness of visualization. In Fig. 14, the two scatterers are located at about 0 - and 6-m heights, with a height difference below the Rayleigh resolution limit and with a velocity of $-3.5 \mathrm{~mm} /$ year. It is apparent that they are separated by the superresolution Diff-Tomo ABF, whereas they collapse into one spectral peak with BF. The peak sidelobe level obtained with $\mathrm{ABF}$ is approximately $5 \mathrm{~dB}$ better than that of BF. In Fig. 15, two scatterers are imaged at -3 and $4 \mathrm{~m}$, with a deformation velocity around $-3 \mathrm{~mm} /$ year. Again, $\mathrm{ABF}$ can separate the two scatterers, while BF cannot. Moreover, in this case, it is apparent that the ABF spectral peaks are sharper than those of BF. It is also worth observing that the superresolved scatterers in these two ABF examples have a slightly different deformation velocity.

Still considering the 30-track Naples data set, to get an indication of the quality of the heights estimated with the automated Diff-Tomo processing, we compared them with the heights extracted via the Tomo-SAR processing described in

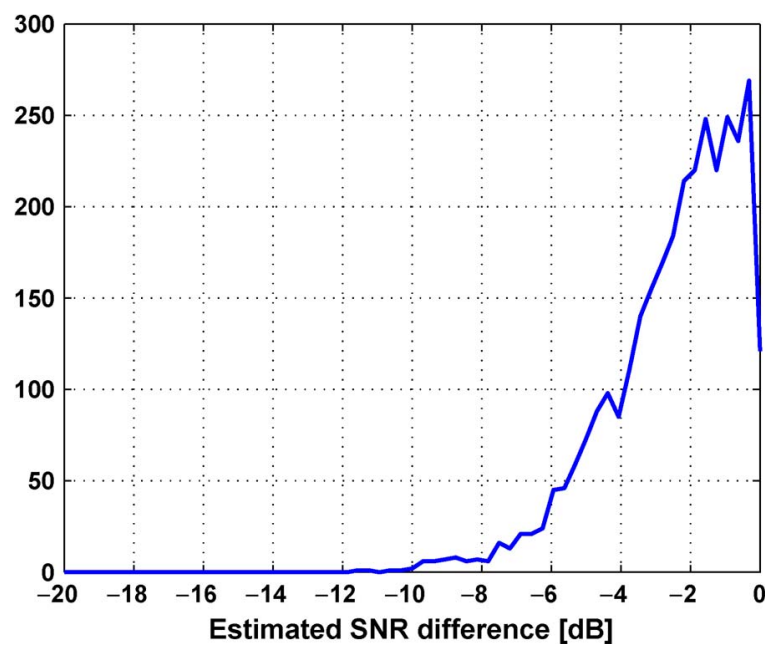

Fig. 17. Histogram of the estimated $S N R$ differences (in decibels) between double scatterers.

TABLE I

Computational Time Obtained in Testing the Presence of One, Two, ANd Three Scatterers in a Cell, Averaged Over the Whole DATA SET

\begin{tabular}{lc}
\hline & Computational time [s] \\
\hline 1 scatterer & 0.11 \\
2 scatterers & 0.23 \\
3 scatterers & 0.34 \\
\hline
\end{tabular}

[25] on the same data set, after the compensation of the phase histories related to the ground deformation [10]. In fact, owing to the experiments in [24] and [25], it is reasonable to assume the Tomo-SAR heights as a benchmark. The correlation coefficients between the estimates in the two frameworks show a good agreement, as they amount to 0.95 for the single-scatterer heights and 0.85 and 0.80 for the higher and the lower heights of the double scatterers. With the same quality check, a very good 
agreement is indeed shown on the Rome data set, for which the height correlation coefficients are equal to 0.98 for single scatterers and 0.95 and 0.91 for the higher and lower heights of the double scatterers. This improvement can be explained with the improved calibration of the Rome data set with respect to the 30-track Naples data set. A further confirmation of the quality of the estimated scatterer heights can be gained by geolocalizing the scatterer coordinates and superimposing them on an ortophotograph. In fact, should the scatterer heights be wrongly estimated, a ground mislocation would result in the geocoded map, provided also that the scattering mostly comes from buildings and infrastructures. The geocoded maps for single and double dominant scatterers in the Rome data set are reported in Fig. 16 and show a good match between identified scatterer locations and ground structures.

Also, the estimated $S \hat{N} R$ differences between double scatterers have been analyzed; the resulting histogram is plotted in Fig. 17. The mean difference is around $2.5 \mathrm{~dB}$, indicating that, typically, the weaker of the scatterer pair is comparable to the dominant in terms of backscattered power.

To complete the analysis of the performance of the proposed algorithm, the computational time has been measured in testing the presence of one, two, and three scatterers in a cell. The obtained values have been averaged over all the range-azimuth cells and reported in Table I. A quasi-linear increase of the computational time with the tested number of scatterers is apparent.

\section{CONCLUSION}

In this paper, a new Diff-Tomo algorithm for multiplescatterer identification in scenarios affected by the layover phenomenon has been proposed and tested with real spaceborne data sets over the cities of Naples and Rome, also validating the capabilities of superresolution adaptive differential tomographic processing. The focus was on single- and doublescatterer detection, height and deformation velocity estimation, and the phenomenological analysis of the results. Indications of the goodness of the information extracted over an extended area have been obtained. Moreover, owing to the superresolution properties of the adaptive processing, we confirmed the possibility to identify double-scattering sources with height difference lower than the Rayleigh limit which affects linear processing. It is worth noting that the proposed algorithm is also able to extract higher order scatterers without significantly increasing the computational burden.

A drawback of the proposed identification algorithm is the need for multilooking to make ABF operative, which reduces the spatial horizontal resolution of the velocity map. Future work will regard the reduction of the multilook degree in the adaptive processing; a first sample result has been reported in [35]. Efforts will also be spent in experimenting a singlelook adaptive Diff-Tomo method, proposed in [36], in order to obtain superresolution height and velocity measures at full horizontal resolution. However, given the very high spatial resolution available with the new generation X-band systems, e.g., TerraSAR-X [15], [18] and COSMO-SkyMed [37], it is expected that it will be possible to reach a satisfactory multilooking degree for a stable covariance matrix inversion in the $\mathrm{ABF}$ focusing without necessarily employing a large multilook cell. Furthermore, concerning the specific aspect of height superresolution, comparisons are in order between the ABF scatterer identification and the compressive-sensing-based method proposed in [18] for Diff-Tomo, which has shown good promise.

\section{ACKNOWLEDGMENT}

The authors would like to thank Dr. G. Fornaro from IREA-CNR (Italy) for furnishing the calibrated real data and the differential SAR interferometry measures for the comparisons, for the useful discussions, and for the support in geocoding.

\section{REFERENCES}

[1] R. Bamler and P. Hartl, "Synthetic aperture radar interferometry," Inverse Probl., vol. 14, no. 4, pp. R1-R54, Aug. 1998.

[2] D. T. Sandwell and E. J. Price, "Phase gradient approach to stacking interferograms," J. Geophys. Res., vol. 103, no. B12, pp. $30183-30204$, 1998.

[3] A. Ferretti, C. Prati, and F. Rocca, "Nonlinear subsidence rate estimation using permanent scatterers in differential SAR interferometry," IEEE Trans. Geosci. Remote Sens., vol. 38, no. 5, pp. 2202-2212, Sep. 2000.

[4] P. Berardino, G. Fornaro, R. Lanari, and E. Sansosti, "A new algorithm for surface deformation monitoring based on small baseline differential SAR interferograms," IEEE Trans. Geosci. Remote Sens., vol. 40, no. 11, pp. 2375-2383, Nov. 2002.

[5] F. Gini, F. Lombardini, and M. Montanari, "Layover solution in multibaseline SAR interferometry," IEEE Trans. Aerosp. Electron. Syst., vol. 38, no. 4, pp. 1344-1356, Oct. 2002.

[6] G. Ferraiuolo, F. Meglio, V. Pascazio, and G. Schirinzi, "DEM reconstruction accuracy in multichannel SAR interferometry," IEEE Trans. Geosci. Remote Sens., vol. 47, no. 1, pp. 191-201, Jan. 2009.

[7] A. Reigber and A. Moreira, "First demonstration of airborne SAR tomography using multibaseline L-band data," IEEE Trans. Geosci. Remote Sens., vol. 38, no. 5, pp. 2142-2152, May 2000.

[8] J. Homer, I. D. Longstaff, Z. She, and D. Gray, "High resolution 3-D imaging via multi-pass SAR," Proc. Inst. Elect. Eng. F-Radar, Sonar Navig., vol. 149, no. 1, pp. 45-50, Feb. 2002.

[9] F. Lombardini and A. Reigber, "Adaptive spectral estimators for multibaseline SAR tomography with airborne L-band data," in Proc. IEEE Int. Geosci. Remote Sens. Symp., Toulouse, France, Jul. 2003, pp. 2014-2016.

[10] G. Fornaro, F. Lombardini, and F. Serafino, "Three-dimensional multipass SAR focusing: Experiments with long-term spaceborne data," IEEE Trans. Geosci. Remote Sens., vol. 43, no. 4, pp. 702-714, Apr. 2005.

[11] S. R. Cloude, "Dual-baseline coherence tomography," IEEE Geosci. Remote Sens. Lett., vol. 4, no. 1, pp. 127-131, Jan. 2007.

[12] F. Lombardini, G. Fornaro, M. Pardini, D. Reale, F. Serafino, F. Soldovieri, and M. Costantini, "SAR tomography for scene elevation and deformation reconstruction: Algorithms and potentialities," in Proc. IEEE Radar Conf., Rome, Italy, May 2008, pp. 1-7.

[13] S. Tebaldini, "Forest SAR tomography: A covariance matching approach," in Proc. IEEE Radar Conf., Rome, Italy, May 2008, pp. 1-6.

[14] S. Sauer, L. Ferro-Famil, A. Reigber, and E. Pottier, "Three-dimensional imaging and scattering mechanism estimation over urban scenes using dual-baseline polarimetric InSAR observations at L-band," IEEE Trans. Geosci. Remote Sens., vol. 49, no. 11, pp. 4616-4629, Nov. 2011.

[15] X. X. Zhu, N. Adam, and R. Bamler, "Space-borne high resolution SAR tomography in urban environment using TerraSAR-X data," in Proc. CEOS SAR Calibration Validation Workshop, Oberpfaffenhofen, Germany, Nov. 2008

[16] S. Tebaldini, "Algebraic synthesis of forest scenarios from multibaseline PolInSAR data," IEEE Trans. Geosci. Remote Sens., vol. 47, no. 12, pp. 4132-4142, Dec. 2009.

[17] A. Budillon, A. Evangelista, and G. Schirinzi, "Three-dimensional SAR focusing from multipass signals using compressive sensing," IEEE Trans. Geosci. Remote Sens., vol. 49, no. 1, pp. 488-499, Jan. 2011. 
[18] X. X. Zhu and R. Bamler, "Tomographic SAR inversion by L1-norm regularization-The compressive sensing approach," IEEE Trans. Geosci. Remote Sens., vol. 48, no. 10, pp. 3839-3846, Oct. 2010.

[19] F. Lombardini, "Differential tomography: A new framework for SAR interferometry," IEEE Trans. Geosci. Remote Sens., vol. 43, no. 1, pp. 3744, Jan. 2005.

[20] A. Ferretti, M. Bianchi, C. Prati, and F. Rocca, "Higher-order permanent scatterers analysis," EURASIP J. Adv. Signal Process., vol. 2005, no. 20, pp. 3231-3242, Dec. 2005.

[21] F. Serafino, F. Soldovieri, F. Lombardini, and G. Fornaro, "Singular value decomposition applied to 4D SAR imaging," in Proc. IEEE Int. Geosci. Remote Sens. Symp., Seoul, South Korea, Jul. 2005, pp. 2701-2704.

[22] G. Fornaro, F. Lombardini, and F. Serafino, "Multidimensional imaging with ERS data," in Proc. ESA FRINGE Workshop, Frascati, Italy, Dec. 2005.

[23] F. Lombardini and G. Fornaro, "First trials of Fourier and adaptive tomoDoppler SAR imaging," in Proc. IEEE Int. Geosci. Remote Sens. Symp., Seoul, South Korea, Jul. 2005, pp. 2656-2659.

[24] F. Lombardini, M. Pardini, G. Fornaro, F. Serafino, L. Verrazzani, and M. Costantini, "Linear and adaptive spaceborne three-dimensional SAR tomography: A comparison on real data," IET Radar, Sonar Navig., vol. 3, no. 4, pp. 424-436, Aug. 2009.

[25] F. Lombardini and M. Pardini, "Detection of scatterer multiplicity in spaceborne SAR tomography with array errors," in Proc. IEEE Radar Conf., Pasadena, CA, May 2009, pp. 1-6.

[26] G. Krieger and A. Moreira, "Multistatic SAR satellite formations: Potentials and challenges," in Proc. IEEE Int. Geosci. Remote Sens. Symp., Seoul, South Korea, Jul. 2005, pp. 2680-2684.

[27] H. L. Van Trees, Optimum Array Processing. Part IV of Detection, Estimation and Modulation Theory. Hoboken, NJ: Wiley, 2002.

[28] A. De Maio, G. Fornaro, and A. Pauciullo, "Detection of single scatterers in multidimensional SAR imaging," IEEE Trans. Geosci. Remote Sens., vol. 47, no. 7, pp. 2284-2297, Jul. 2009.

[29] F. Lombardini and F. Gini, "Model order selection in multibaseline interferometric radar systems," EURASIP J. Adv. Signal Process., vol. 2005, no. 20, pp. 3206-3219, Dec. 2005.

[30] F. Bordoni, F. Gini, and M. Pardini, "Capon-LS for model order selection of interferometric SAR signals corrupted by multiplicative noise," in Proc. 4th Int. Symp. Signal Process. Inf. Technol., Rome, Italy, Dec. 2004, pp. 171-174.

[31] G. Fornaro and F. Serafino, "Imaging single and double scatterers in urban areas via SAR tomography," IEEE Trans. Geosci. Remote Sens., vol. 44, no. 12, pp. 3497-3505, Dec. 2006.

[32] G. Fornaro, F. Serafino, and D. Reale, "4D SAR imaging for height estimation and monitoring of single and double scatterers," IEEE Trans. Geosci. Remote Sens., vol. 47, no. 1, pp. 224-237, Jan. 2009

[33] F. Lombardini, M. Montanari, and F. Gini, "Reflecitvity estimation for multibaseline interferometric radar imaging for layover extended sources," IEEE Trans. Signal Process., vol. 51, no. 6, pp. 1508-1519, Jun. 2003

[34] G. Fornaro, A. Pauciullo, and F. Serafino, "Deformation monitoring over large areas with multipass differential SAR interferometry: A new approach based on the use of spatial differences," Int. J. Remote Sens., vol. 30, no. 6, pp. 1455-1478, Jun. 2009.

[35] F. Lombardini and M. Pardini, "Multiple scatterers identification in complex scenarios with adaptive differential tomography," in Proc. IEEE Int. Geosci. Remote Sens. Symp., Cape Town, South Africa, Jul. 2009, pp. III-908-III-911.

[36] F. Lombardini, "Full-resolution adaptive differential tomography," in Proc. IEEE Int. Geosci. Remote Sens. Symp., Cape Town, South Africa, Jul. 2009, pp. III-176-III-179.

[37] S. Mezzasoma, A. Gallon, F. Impagnatiello, G. Angino, S. Fagioli, A. Capuzi, F. Caltagiorne, R. Leonardi, and I. Ziliotto, "COSMO-SkyMed system commissioning: End-to-end system performance verification," in Proc. IEEE Radar Conf., Rome, Italy, May 2008, pp. 1-5.

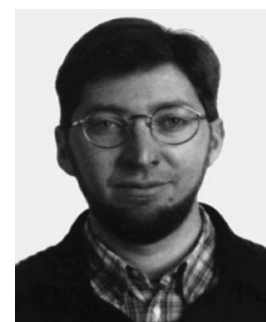

Fabrizio Lombardini (M'93-SM'03) received the Italian Laurea degree (with honors) in electronic engineering and the $\mathrm{Ph} . \mathrm{D}$. degree in telecommunication engineering from the University of Pisa, Pisa, Italy, in 1993 and 1997, respectively.

From 1998 to 1999 , he was a Postdoctoral Researcher with the Department of Electronic and Electrical Engineering, University College London, London, U.K. Then, he joined the Department of Information Engineering, University of Pisa, where he is currently an Assistant Professor. He has given lectures at universities and institutions in Italy and abroad and has been a Guest Coeditor of the European Association for Signal Processing (EURASIP) Journal of Applied Signal Processing Special Issue on Advances in Interferometric SAR Processing. His general interests include the areas of statistical signal processing; estimation and detection; adaptive, superresolution, and robust spectral analysis; array processing; irregular sampling; multiplicative noise; and performance bound evaluation, with application to radar systems and synthetic aperture radar (SAR) environmental remote sensing. In particular, his research interests include multibaseline and multifrequency interferometric SAR algorithms and systems (both cross and along tracks), 3-D SAR tomography, differential SAR interferometry, multisensor data fusion, radar detection in non-Gaussian clutter, and, recently, localization in radiofrequency identification systems (RFID). He has been the originator of the new interferometric mode of 4-D differential SAR tomography.

Dr. Lombardini was a recipient of the European Union Marie Curie Fellowship of the Training and Mobility of Researchers Program. He has chaired ten special sessions on SAR multibaseline/multichannel interferometry/3-D techniques at international conferences. He has been a Lecturer or a Colecturer of four tutorials on SAR tomography and multidimensional SAR imaging at the European Conference on Synthetic Aperture Radar (EUSAR'08), IEEE Radar Conference (RadarCon09), IEEE International Geoscience And Remote Sensing Symposium (IGARSS'10), and IGARSS'11.

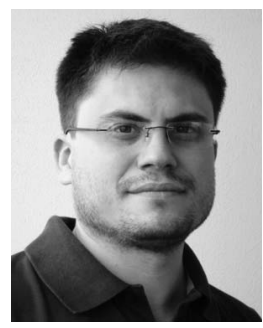

Matteo Pardini (S'02-M'10) received the M.Sc. degree (cum laude) in telecommunication engineering and the Ph.D. degree in information engineering, with research on "Interferometric, Multidimensional, and Distributed Sensing Techniques," from the University of Pisa, Pisa, Italy, in 2006 and 2010, respectively.

In July 2006, he joined the Department of Information Engineering, University of Pisa, as a Research Assistant. Since January 2010, he has been a Research Scientist with the Microwaves and Radar Institute, German Aerospace Center (DLR), Oberpfaffenhofen, Germany, after a visiting period from August to December 2009. His general interests include the area of radar signal processing, with particular emphasis on parameter estimation theory, detection, spectral estimation, performance bound evaluation, and array processing. In particular, his research activity is focused on the coherent processing of synthetic aperture radar data for information extraction, tomography, and differential tomography. He regularly acts as a Reviewer for the IET Radar, Sonar \& Navigation, the IET Electronic Letters, the Elsevier Signal Processing, and the Springer Journal on Advances in Signal Processing.

Dr. Pardini has been a member of the EURASIP since 2006. In 2007, he was a recipient of one of the five "Best Remote Sensing Thesis Awards" from the IEEE Geoscience and Remote Sensing South Italy Chapter, and in 2009, he was also a recipient of the "Best Paper Award Francesco Carassa" from the Italian Associazione Gruppo Telecomunicazioni e Tecnologie dell'Informazione, for the paper presented at the Remote Sensing Scientific Session of the 2009 General Assembly. He regularly acts as a Reviewer for the IEEE TRANSACTIONS on Geoscience and Remote SENSING and the IEEE TRANSACtions on SignAl PROCESSING. 